\title{
Sternal transplant using cadaveric allograft: quantitative and qualitative assessment of bone healing by computed tomography
}

\author{
Chiara Giraudo $^{1}$, Francesca Nistri ${ }^{1}$, Pia Ferrigno ${ }^{2}$, Giampiero Dolci ${ }^{3}$, Roberto Stramare ${ }^{1}$, Giuseppe Guglielmi ${ }^{4}$, \\ Marco Mammana ${ }^{2}$, Emilio Quaia ${ }^{1}$, Domenica Giunta ${ }^{3}$, Andrea Dell'Amore ${ }^{2}$, Federico Rea ${ }^{2}$ \\ ${ }^{1}$ Radiology Institute, Department of Medicine - DIMED, University of Padova, Padova, Italy; ${ }^{2}$ Thoracic Surgery Unit, Department of \\ Cardiothoracic Surgery and Vascular Sciences, University of Padova, Padova, Italy; ${ }^{3}$ Department of Cardiothoracic Surgery, S. Orsola Malpighi \\ University Hospital, Bologna, Italy; ${ }^{4}$ Department of Clinical and Experimental Medicine, Foggia University School of Medicine, Foggia, Italy \\ Correspondence to: Giuseppe Guglielmi. Department of Clinical and Experimental Medicine, Foggia University School of Medicine, Viale L. Pinto, 1, \\ 71121 Foggia, Italy. Email: giuseppe.guglielmi@unifg.it.
}

Background: Sternal transplant using cadaveric allograft (STCA) is a complex and rarely performed surgical procedure usually applied for massive bone tissue loss, sternotomy complications, or neoplastic resections. Although radiological imaging and especially computed tomography (CT) is routinely applied for the post-surgical assessment, up to now, a standardized approach evaluating the outcome of STCAs is missing. Therefore, aim of this study was to qualitatively and quantitatively evaluate, by CT, bone healing after STCA.

Methods: The first and the last available postsurgical CT of patients who underwent STCA in two tertiary centers between 2009 and 2017 were collected. Standardized regions of interest were applied on the cancellous bone along the transplanted sternum, and, as reference, on the fourth thoracic vertebra, at both time points, collecting the density values. The areas nearby the fixation devices were assessed by a fourpoints qualitative score. To evaluate the mineralization, the analysis of the variance (ANOVA) with post-hoc Bonferroni correction was applied for the quantitative measurements while the Wilcoxon test was used for the qualitative score $(\mathrm{P}<0.05)$. To evaluate the intra-rater reliability of the qualitative and the quantitative analyses, the same rater repeated the measurements after two months and the Cohen's kappa (k) and the intraclass correlation coefficient (ICC) were computed.

Results: Fourteen patients (11 females, $61 \pm 12.8$ years) were examined. The first control CTs were performed $32 \pm 40.26$ days after the STCA and the last CT were acquired after $729 \pm 745$ days. The quantitative and the qualitative score significantly increased between the two intervals $(\mathrm{P}<0.05$, each). The density of the transplanted sternum was lower than that of the vertebral reference at the first CT $(\mathrm{P}=0.006)$ while no differences occurred at the last control $(\mathrm{P}=0.361)$. The assessments showed high intra-rater reliability and agreement (ICC $\geq 0.890, \mathrm{k} \geq 0.906$ ).

Conclusions: The hereby-proposed qualitative and quantitative methods demonstrated to be good tools for assessing bone healing after STCA.

Keywords: Bone transplantation; computed tomography (CT); imaging; thoracic surgery

Submitted Jan 10, 2020. Accepted for publication Sep 03, 2020.

doi: $10.21037 /$ qims-20-90

View this article at: http://dx.doi.org/10.21037/qims-20-90 


\section{Introduction}

In thoracic and cardiac surgery, sternal resection might be necessary because of tumors, infections, and surgical dehiscence (1-3). Recently, the use of bone grafts for chest wall reconstruction provided excellent results $(2,4,5)$. Bone grafts promoting new bone formation and healing can be of three main types: auto-grafts (grafts from the host bone stock), allografts (grafts from cadaveric bone stock), and synthetic bone graft substitutes (6). Allografts provide an osteoconductive matrix that, surrounded by granulation tissue, can undergo vascular and osteogenic cell invasion $(6,7)$.

In particular, sternal transplant using cadaveric allograft (STCA) is a complex and rarely performed surgical procedure usually applied for massive bone tissue loss, sternotomy complications, or neoplastic resections $(4,5,8)$.

Indeed, with this technique the chest wall function can be re-established with a minimal risk of graft rejection $(1,3,4,5,8)$. The absence of vital marrow cells reduces the occurrence of immunogenetic graft complications and the cryopreserved tissue is associated with a lower risk of infections $(2,3)$.

Although radiological imaging and especially computed tomography (CT) is routinely applied for the post-surgical thoracic assessment, up to now, a standardized approach evaluating the outcome of STCAs is missing. Tailored scoring systems have been proposed in similar fields, as to investigate sternal healing after sternotomy in patients who underwent cardiac surgery, but not for STCA (9). Indeed, the current literature on the use of CT for SCTA has been mostly based on anecdotal evidence, mainly deriving from case reports or series, and its focused on post-surgical complications $(4,5)$. In fact, CT nicely demonstrates subtle erosions, periosteal reaction, sclerosis, and swelling in the soft tissue (10). For instance, Marulli et al. (1), in one of the largest studies, longitudinally assessed by CTs eighteen patients evaluating the occurrence of dehiscence, infections, or confirming the correct positioning of the graft.

Considering the clinical impact of STCA and its expected increasing application, radiologists should provide a precise and accurate assessment of the repairing process related to this surgical procedure. With this purpose, it should be highlighted that CT can also provide quantitative information regarding bone density. It has been demonstrated that such quantitative data not only correlate with bone mineral density in patients with osteoporosis $(11,12)$ but also that they provide important information regarding the bone repair process. In particular, it has been shown that bone repairing is characterized by a progressive increase of density at CT $(13,14)$.

Thus, aim of this study was to propose a qualitative and a quantitative CT-based method to evaluate bone healing after STCA.

\section{Methods}

\section{Study design}

All patients who underwent STCA in two tertiary centers (i.e., Thoracic Surgery of the Padova University Hospital and Thoracic Unit of the University Hospital of Bologna) between 2009 and 2017 were retrospectively evaluated for this study which has been approved by the Institution Review Board (PD00-19-03CT). Written informed consent was obtained from the patients for publication of this manuscript and any accompanying images. Among the patients who underwent STCA, only those satisfying the following criteria were included: (I) availability of at least two CTs after the STCA; (II) CT examinations without contrast injection; (III) slice thickness of $1-3 \mathrm{~mm}$. In case of multiple post-surgical CT examinations, the first and the last available scans were selected.

\section{Surgical technique}

The applied surgical technique refers to previously published methods $(1,2,15)$. In particular, sternochondral blocks were surgically removed from multi-organ donors according to the current legislation. Aiming to sterilize the samples and suppress the immunogenic activity, the allografts were immersed in a sterile saline solution with antibiotics for 72 hours at $+4{ }^{\circ} \mathrm{C}$ and then irradiated, packed, and stored at $-80^{\circ} \mathrm{C}$. Applying this process, not only red blood cell and/or human leukocyte antigen compatibility are not necessary but there is also no need for immunosuppressive therapy after the transplant. Prior to the implantation, under general anesthesia, the graft was unwrapped and immersed in a sterile solution $(0.9 \%$ sodium chloride).

According to the different diseases which led to the transplant, a full thickness partial or complete sternectomy $e n-b l o c$, including costal cartilages, was performed. The allograft was then modeled avoiding any overlapping or misalignment. To guarantee the appropriate stability of the implant, care was taken in reducing the space between the 
thoracic cage of the recipient and the sternum of the donor. To prevent any dislocation, the allograft was then fixed with titanium bars and screws (Synthes, Solothurn, Switzerland) avoiding costal cartilages. For partial sternectomies the sternal allograft was fixed to the residual sternum of the recipient (Figure 1). At the end of the procedure, major

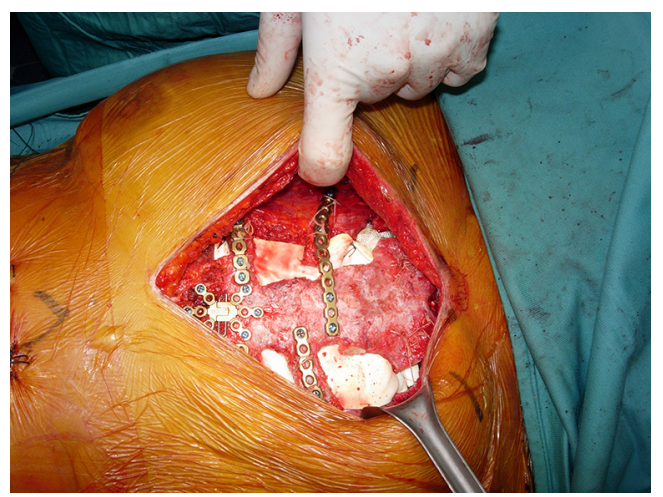

Figure 1 Sternal transplant with replacement of the manubrium and of the body fixed with titanium bars and screws in a 59 years old female patient affected by sternal metastasis from breast carcinoma. pectoralis muscle flaps were applied to cover the implant.

\section{CT-based quantitative and qualitative assessment}

One radiologist with 4 years of experience in thoracic imaging, blinded to all clinical data, performed all quantitative and qualitative evaluations.

Regarding the quantitative measurements, a standardized round region of interest (ROI) of $30 \mathrm{~mm}^{2}$ was applied on multiple areas of cancellous bone along the transplanted sternum (i.e., respectively seven ROIs in case of transplant of the entire sternal body with the manubrium, five ROIs if only the body was used, and two ROIs if the manubrium with the upper part of the body was transplanted) on each side, for each patient, at both time points (Figure 2).

Aiming to standardize the measurements at each time point, the seven examined areas were chosen on the sagittal plane applying a constant interval of $15 \mathrm{~mm}$ between the subsequent ROIs and using the costosternal joints as reference. The density value, expressed in Hounsfield unit (HU) was collected from each performed ROI.

The same-size ROI was also applied on the cancellous bone of the fourth thoracic vertebra (T4) and the extracted

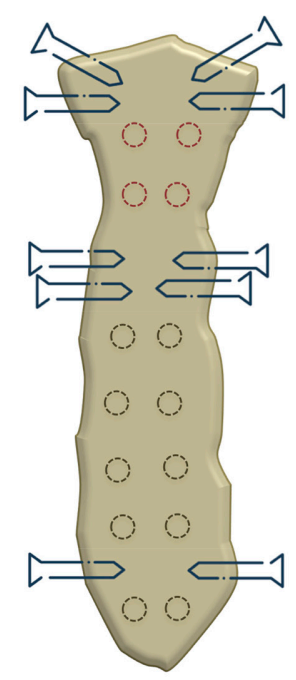

3 $30 \mathrm{~mm}^{2}$ regions of interest for the quantitative assessment in case of sternal transplant of the manubrium and of the upper body
$30 \mathrm{~mm}^{2}$ regions of interest for the quantitative assessment in patients with sternal transplant of the body and the manubrium
$30 \mathrm{~mm}^{2}$ regions of interest for the quantitative assessment in patients with sternal transplant of the body

Figure 2 Graphic representation demonstrating the areas of the computed tomography-based quantitative and qualitative measurements. 
$\mathrm{HU}$ values were used as reference.

To avoid biases due to beam hardening artifacts caused by the metal component of screws and plates, the areas close to the fixation devices were excluded from the quantitative measurements. Nevertheless, on such areas a modified version of the Stacy score for bone healing (absent, low, moderate, and complete healing) was applied as a qualitative score (9).

\section{Statistical analysis}

Descriptive statistics were applied for demographics and clinical categorical data.

To evaluate if differences in the HU values of the cancellous bone occurred between the first and the last available post-surgical CT and if they differed from the bone area selected as reference (i.e., cancellous bone of T4), the analysis of the variance (ANOVA) test with post-hoc Bonferroni correction was applied.

The Wilcoxon test was used to compare the results of the qualitative score between the two time points. The applied level of significance for all statistical analyses was $\mathrm{P}<0.05$.

To assess the intra-observer reliability of the proposed methods, after 2 months, the same rater repeated all the evaluations and the Cohen's kappa and the intraclass correlation coefficient (ICC) were computed for the qualitative and the quantitative analyses, respectively. ICC values above 0.750 were considered excellent (16).

All statistical analyses were performed using SPSS Statistics 25.0 (IBM Corp, Armonk, NY, USA).

\section{Results}

\section{Surgical procedure and outcome}

Among the seventeen patients who underwent STCA in the two centers during the selected interval of time, fourteen [11 females, with a mean age \pm standard deviation (SD) at surgery of $61 \pm 12.8$ years] matched the inclusion criteria.

STCA was performed because of sternal chondrosarcoma in six patients, sternal metastasis from breast carcinoma and previous surgical dehiscence in three patients each, neuroendocrine tumor and plasmacytoma in one patient each. Eight patients underwent transplant of the sternal body, three of the body and the manubrium and other three of the manubrium with the upper portion of the body.

No short- or long-term post-surgical complications were reported.

\section{Quantitative and qualitative CT assessment}

Six patients underwent four CT scans after the STCA, five had three examinations and three only two controls. The interval of time between the STCA and the first CT was of $32 \pm 40.26$ while an interval of $729 \pm 745$ days occurred between the transplant and the last available CT.

The mean HU values of the transplanted sternal bone significantly increased between the first and the last available post-surgical CT $(118 \pm 35$ vs. $191 \pm 44 \mathrm{HU}$; $\mathrm{P}=0.004$ ) (Figure 3).

Furthermore, the mean HU values of the cancellous bone of the transplanted sternum were significantly lower than those extracted from $\mathrm{T} 4$ at the first CT after the transplant $(\mathrm{P}=0.006)$ while no differences comparing the sternal and the vertebral ROIs at the last available examination occurred $(\mathrm{P}=0.361)$ (Table 1).

Furthermore, the HU values extracted from T4 did not differ between the two time points $(\mathrm{P}=1.000)$ (Figure 4).

The modified version of the Stacy score was significantly higher at the last available post-surgical CTs (mean score $1.2 \pm 0.8$ vs. $2.1 \pm 0.8$ at the first and last available CT, respectively; $\mathrm{P}=0.000$ ) (Figure 5).

Both types of assessment showed a high intra-rater reliability for the quantitative measurements (ICC $=0.990$ and ICC $=0.890$ for the evaluations at the first and last available CT, respectively) and high agreement for the qualitative score ( $\mathrm{k}=0.931$ and $\mathrm{k}=0.906$ for the evaluations at the first and last available CT, respectively).

\section{Discussion}

Our results demonstrated that CT can be successfully applied to evaluate the bone healing process in patients who underwent STCA. In particular, regarding the quantitative evaluation, we are in line with previous studies, even if focused on other surgical procedures, which showed that bone repairing is characterized by a progressive increase of bone density at CT $(13,14)$. Nevertheless, controversial results have also been reported in the literature. For instance, examining the changes occurring in the trabecular bone at fractures sites (i.e., distal radius) Lynch and colleagues (17) observed either an increase or a rise and then a decline of density values within an interval of 12 weeks. This observed difference might be related to the fact that they investigated a different target population, used a diverse time interval, and that we applied bone grafts which enhance the healing process (18). Regarding 

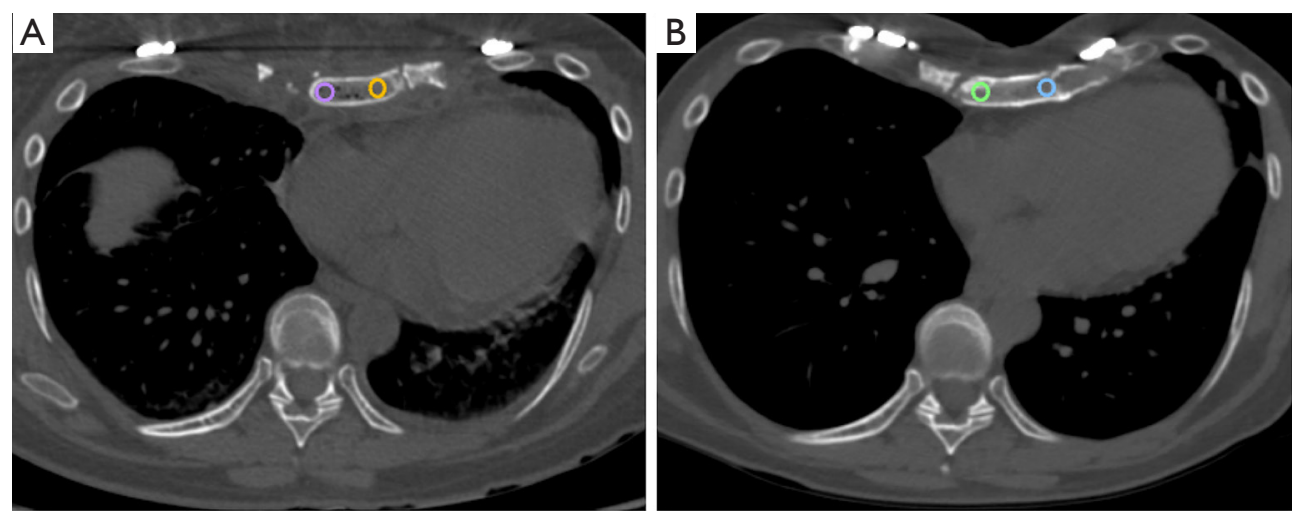

Figure 3 Axial CT images of a 40-year-old female patient examined 15 days (A) and 18 months (B) after the transplant showing one pair of ROIs applied in the cancellous bone, at the same level at each time point [circles in (A) and (B)]. The HU values in the hereby-represented ROIs increased between the two controls [i.e., 72 and $106 \mathrm{HU}$ in (A) and 301 and $278 \mathrm{HU}$ in (B), respectively] demonstrating an increased mineralization.

Table 1 Results of the quantitative computed tomography-based analyses

\begin{tabular}{|c|c|c|c|c|c|c|c|c|c|}
\hline & $\begin{array}{c}\text { Sternal ROI } \\
\text { measurements at } \\
\text { the first CT after } \\
\text { STCA }\left(C T^{1}\right)\end{array}$ & $\begin{array}{c}\text { Sternal ROI } \\
\text { measurements at } \\
\text { the last available CT } \\
\text { after STCA }\left(\mathrm{CT}^{2}\right)\end{array}$ & $\begin{array}{c}\text { Measurement at } \\
\text { the T4 level at } \\
\text { the first CT after } \\
\text { STCA }\left(T 4^{1}\right)\end{array}$ & $\begin{array}{l}\text { Measurement at } \\
\text { the T4 level at the } \\
\text { last available CT } \\
\text { after STCA }\left(\mathrm{T}^{2}\right)\end{array}$ & $\begin{array}{l}\text { One-way } \\
\text { ANOVA(P) }\end{array}$ & $\mathrm{CT}^{1}$ vs. $\mathrm{CT}^{2}$ & $\mathrm{CT}^{1}$ vs. $\mathrm{T} 4^{1}$ & $\mathrm{CT}^{2}$ vs. T4 ${ }^{2}$ & $\mathrm{~T} 4^{1}$ vs. $\mathrm{T} 4^{2}$ \\
\hline $\begin{array}{l}\text { HU value } \\
(\text { mean } \pm \mathrm{SD})\end{array}$ & $118 \pm 35$ & $191 \pm 44$ & $156 \pm 36$ & $155 \pm 33$ & 0.002 & $0.004^{a}$ & $0.006^{a}$ & $0.361^{a}$ & $1.000^{\mathrm{a}}$ \\
\hline
\end{tabular}

a , post-hoc Bonferroni correction; applied level of significance $\mathrm{P}<0.05$. CT, computed tomography; HU, Hounsfield unit; SD, standard deviation; $\mathrm{T} 4, \mathrm{HU}$ values extracted from the cancellous bone of the fourth thoracic vertebra; ROI, region of interest of $30 \mathrm{~mm}^{2}$ in the cancellous bone of the transplanted sternum (see Figure 3). STCA, sternal transplant using cadaveric allograft.
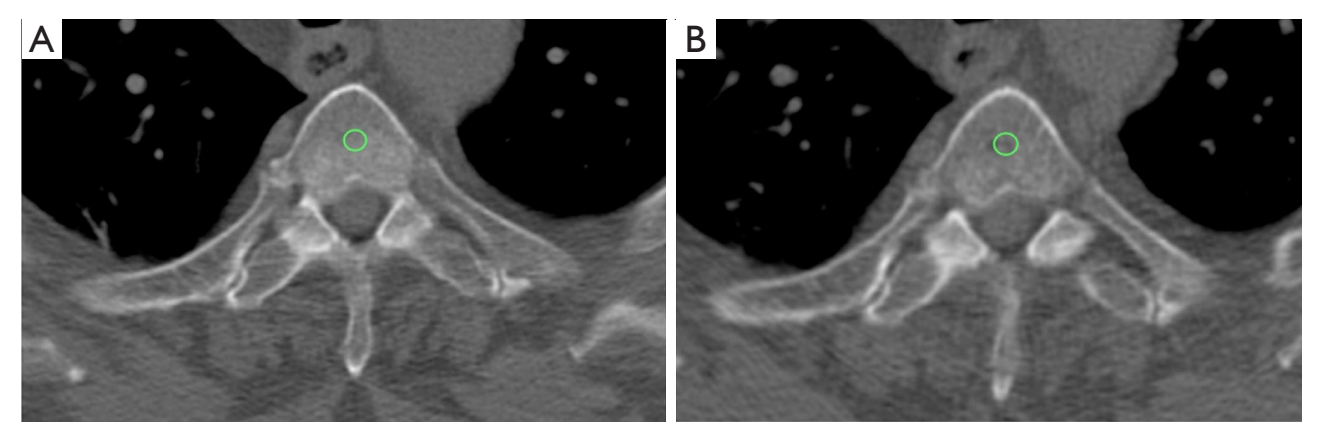

Figure 4 Axial CT images of a 78-year-old male examined 55 days (A) and 16 months (B) after the sternal transplant showing the ROIs applied on the fourth thoracic vertebral body, used as reference [green circles in (A) and (B)] which provided almost identical HU values at both time points [i.e., $231 \mathrm{HU}$ in (A) and $211 \mathrm{HU}$ in (B), respectively].

the integration of bone grafts, different mechanisms, like osteoinduction, osteoconduction, and osseointegration have to be addressed (19). Osteoinduction activates the osteogenesis and it occurs in any bone healing process. Osteoconduction is characterized by the bone growth on a surface and it is hampered by implant materials with low biocompatibility (e.g., copper, silver). Osseointegration is really crucial since it reflects the firm fixation of the implanted sternum obtained by bone to implant contact (20).

It is very challenging to demonstrate the cellular 

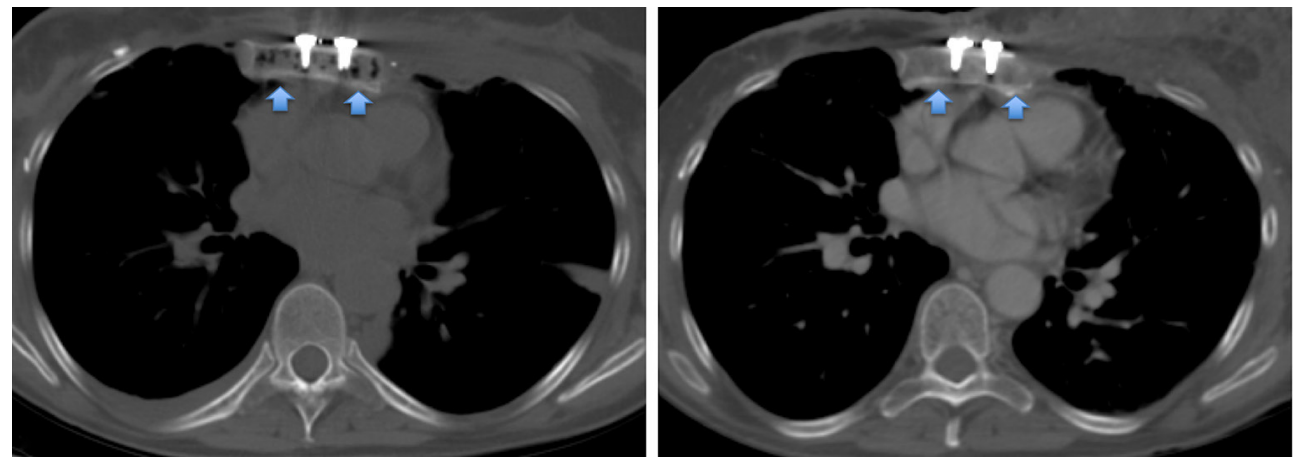

Figure 5 Axial CT images of a 72-year-old female patient examined 17 days (A) and 9 months (B) after the sternal transplant demonstrating an increased mineralization close to the surgical screws [blue arrows; mean qualitative score of 1.5 in (A) and 2.5 in (B)].

integration of bone tissue in the sternal graft since the collection of bioptic samples raise ethical concerns $(15,21)$. Our study demonstrates that not only CT-based quantitative analyses but also a qualitative assessment can be successfully used to monitor and characterize the bone healing process after sternal graft positioning.

Indeed, regarding the qualitative evaluation, the herebyapplied modified version of the score proposed by Stacy et al. (9) for bone repair after sternotomy turned out to be a reliable method also for patients who underwent STCA.

Even if STCAs can be assessed with CT, it should not be overlooked that post-surgical imaging has to deal with some challenges like, for instance, the presence of artifacts due to metal implants and devices. Ferreira et al. (22) examining patients with bone grafting for shoulder arthroplasty highlighted that metal artifacts may give the appearance of graft healing and considered CT inappropriate for the detection of bone graft resorption. In our patients, the observed increase in the qualitative scores at the second time point and the overall good clinical outcome let us assume that there was not a massive tissue resorption which has been overlooked. Moreover, the high agreement of the measurements suggests a good reproducibility of the method, although it has to be further tested on a larger population including inter-rater evaluations.

Despite our promising results and the fact that CT is often the main technique applied in the post-surgical setting also for long-term follow-ups, other diagnostic tools could be used for monitoring STCA patients. Indeed, molecular imaging could also provide crucial information about bone viability $(3,4)$. Kaláb et al. (3) and Dell'Amore et al. (4) successfully used Technetium $99 \mathrm{~m}$-bisphosphonate to monitor osteoblast metabolism and osteoblastic activity after allogenous sternal bone graft. Studies comparing the diagnostic value of radiological, nuclear and hybrid imaging could contribute to the development of appropriate diagnostic criteria and guidelines in this particular group of patients.

Some limitations of this study have to be considered. First of all, the retrospective study design did not allow a precise standardization of the time interval between the two CT scans. In particular, since most of our population was composed of cancer patients and none had complications after the STCA, the CTs at follow-up were mainly performed as part of the oncology protocol. Despite such heterogeneity, our results demonstrated that after an average interval of two years a complete healing is achieved. The development of specific guidelines, considering several factors like the disease that led to the STCA, patients' comorbidities, and radiation safety principles, are necessary to suggest the appropriate timing of the follow-up.

Despite the high intra-rater reproducibility of the hereby proposed methods, it might be argued that especially for the quantitative assessment, its application in the clinical practice might be hampered by time constraints. Future studies applying automatic segmentations, which already provided good results for investigating the level of osteointegration and osteogenesis after orthopedic and maxillo-facial surgery (23), or automated methods of quantification $(24,25)$ should be performed.

Lastly, as previously mentioned, the small sample size should be addressed, even if the limited amount of cases is surely related to the rarity of the surgical procedure. International multicenter studies may overcome this drawback and further assess the diagnostic value of our scoring systems. 


\section{Conclusions}

In conclusion, the hereby-proposed CT-based qualitative and quantitative methods demonstrated to be good tools for the assessment of bone healing in patients who underwent STCA. Further research in this field with tailored prospective studies, including a larger population, may even lead to a model predicting the repairing process.

\section{Acknowledgments}

Funding: None.

\section{Footnote}

Conflicts of Interest: All authors have completed the ICMJE uniform disclosure form (available at http://dx.doi. org/10.21037/qims-20-90). Prof. GG serves as an unpaid editorial board member of Quantitative Imaging in Medicine and Surgery. The other authors have no conflicts of interest to declare.

Ethical Statement: The study was approved by the Institution Review Board (PD00-19-03CT). Written informed consent was obtained from the patients for publication of this manuscript and any accompanying images.

Open Access Statement: This is an Open Access article distributed in accordance with the Creative Commons Attribution-NonCommercial-NoDerivs 4.0 International License (CC BY-NC-ND 4.0), which permits the noncommercial replication and distribution of the article with the strict proviso that no changes or edits are made and the original work is properly cited (including links to both the formal publication through the relevant DOI and the license). See: https://creativecommons.org/licenses/by-nc-nd/4.0/.

\section{References}

1. Marulli G, Dell'Amore A, Calabrese F, Schiavon M, Daddi N, Dolci G, Stella F, Rea F. Safety and effectiveness of cadaveric allograft sternochondral replacement after sternectomy: a new tool for the reconstruction of anterior chest wall. Ann Thorac Surg 2017;103:898-905.

2. Marulli G, Hamad AM, Cogliati E, Breda C, Zuin A, Rea F. Allograft sterno-chondral replacement after resection of large sternal chondrosarcoma. J Thorac Cardiovasc Surg 2010;139:e69-70.
3. Kaláb M, Karkoška J, Kamínek M, Šantavý P. Successful three-year outcome in a patient with allogenous sternal bone graft in the treatment of massive post-sternotomy defects. Int J Surg Case Rep 2015;7C:6-9.

4. Dell'Amore A, Campisi A, Giunta A, Congiu S, Dolci G, Murana G, Martin Suarez S, Daddi N. Surgical options to treat massive sternal defect after failed Robicsek procedure. J Thorac Dis 2018;10:E410-15.

5. Dell'Amore A, Cassanelli N, Dolci G, Stella F. An alternative technique for anterior chest wall reconstruction: the sternal allograft transplantation. Interact Cardiovasc Thorac Surg 2012;15:944-7.

6. Beaman FD, Bancroft LW, Peterson JJ, Kransdorf MJ, Menk DM, DeOrio JK. Imaging Characteristics of Bone Graft Materials. Radiographics 2006;26:373-88.

7. Carone L, Danesino GM, Pietrobon L, Corsico A, Bonardi M, Sammarchi L, Zappoli F. Bone healing: radiology of normal and diseased bone. ESR Epos 2017. Available online: http://dx.doi.org/10.1594/ecr2017/C-1118

8. Stella F, Dell'Amore A, Dolci G, Cassanelli N, Caroli G, Zamagni C, Bini A. Allogenic sternal transplant after sternectomy for metastasis of ovarian carcinoma. Ann Thorac Surg 2012;93:e71-2.

9. Stacy GS, Ahmed O, Richardson A, Hatcher BM, MacMahon H, Raman J. Evaluation of Sternal Bone Healing with Computed Tomography and a Quantitative Scoring Algorithm. Open Med Imaging J 2014;8:29-35.

10. Li AE, Fishman EK. Evaluation of complications after sternotomy using single- and multi-detector CT with three-dimensional volume rendering. AJR Am J Roentgenol 2003;181:1065-70.

11. Schreiber JJ, Anderson PA, Hsu WK. Use of computed tomography for assessing bone mineral density. Neurosurg Focus 2014;37:E4.

12. Pickhardt PJ, Lee LJ, Munoz del Rio A, Lauder T, Bruce RJ, Summers RM, Pooler BD, Binkley N. Simultaneous screening for osteoporosis at CT colonography: bone mineral density assessment using MDCT attenuation techniques compared with the DXA reference standard. J Bone Miner Res 2011;26:2194-203.

13. Spruit M, Meijers H, Obradov M, Anderson PG. CT Density Measurement of Bone Graft Within an Intervertebral Lumbar Cage: Increase of Hounsfield Units as an Indicator for Increasing Bone Mineral Content. J Spinal Disord Tech 2004;17:232-35.

14. Grigoryan M, Lynch JA, Fierlinger AL, Guermazi A, Fan B, MacLean DB, MacLean A, Genant HK. Quantitative and qualitative assessment of closed fracture healing using 
computed tomography and conventional radiography. Acad Radiol 2003;10:1267-73.

15. Dell'Amore A, Comacchio G, Ferrigno P, Pangoni A, Schiavon M, Rea F. Sternal allograft transplantation for anterior chest wall reconstruction after sternectomy. Multimed Man Cardiothorac Surg 2019. doi: 10.1510/ mmcts.2019.020.

16. Cicchetti DV. Guidelines, criteria, and rules of thumb for evaluating normed and standardized assessment instruments in psychology. Psychol Assess 1994;6:284-90.

17. Lynch JA, Grigoryan M, Fierlinger A, Guermazi A, Zaim S, MacLean DB, Genant HK. Measurement of changes in trabecular bone at fracture sites using X-ray CT and automated image registration and processing. J Orthop Res 2004;22:362-7.

18. Roberts TT, Rosenbaum AJ. Bone graft, bone substitutes and orthobiologics. The bridge between basic science and clinical advancements in fracture healing. Organogenesis 2012;8:114-24.

19. Albrektsson T, Johansson C. Osteoinduction, osteoconduction and osseointegration. Eur Spine J 2001;10:S96-S101.

20. LeGeros RZ. Properties of osteoconductive biomaterials:

Cite this article as: Giraudo C, Nistri F, Ferrigno P, Dolci G, Stramare R, Guglielmi G, Mammana M, Quaia E, Giunta D, Dell'Amore A, Rea F. Sternal transplant using cadaveric allograft: quantitative and qualitative assessment of bone healing by computed tomography. Quant Imaging Med Surg 2021;11(2):502-509. doi: 10.21037/qims-20-90 calcium phosphates. Clin Orthop 2002;395:81-98.

21. Schmidle G, Ebner HL, Klauser AS, Fritz J, Arora R, Gabl M. Correlation of CT imaging and histology to guide bone graft selection in scaphoid non-union surgery. Arch Orthop Trauma Surg 2018;138:1395-405.

22. Ferreira LM, Knowles NK, Richmond DN, Athwal GS. Effectiveness of CT for the detection of glenoid bone graft resorption following reverse shoulder arthroplasty. Orthop Traumatol Surg Res 2015;101:427-30.

23. Grottoli CF, Ferracini R, Compagno M, Tombolesi A, Rampado O, Pilone L, Bistolfi A, Borrè A, Cingolani A, Perale G. A Radiological Approach to Evaluate Bone Graft Integration in Reconstructive Surgeries. Appl Sci 2019;9:1469.

24. Porter SM, Dailey HL, Hollar KA, Klein K, Harty JA, Lujan TJ. Automated Measurement of Fracture Callus in Radiographs Using Portable Software. J Orthop Res 2016;34:1224-33.

25. Nemecek E, Chiari C, Valentinitsch A, Kainberger F, Hobusch G, Kolb A, Hirtler L, Trost C, Vukicevic S, Windhager R. Analysis and quantification of bone healing after open wedge high tibial osteotomy. Wien Klin Wochenschr 2019;131:587-98. 